\title{
Intuitionistic fuzzy modal operators of second type over interval-valued intuitionistic fuzzy sets. Part 1
}

\author{
Krassimir Atanassov \\ Bioinformatics and Mathematical Modelling Dept., Institute of Biophysics \\ and Biomedical Engineering, Bulgarian Academy of Sciences \\ 105 Acad. G. Bonchev Str., 1113 Sofia, Bulgaria, \\ and \\ Intelligent Systems Laboratory \\ Prof. Asen Zlatarov University, 8010 Burgas, Bulgaria \\ e-mail: krat@bas.bg
}

Received: 2 April 2018

Accepted: 20 April 2018

\begin{abstract}
The basic definitions of the concept of interval-valued intuitionistic fuzzy set and of the operations, relations and operators over it are given. Some of its most important applications are described. Ideas for future development of the theory of interval-valued intuitionistic fuzzy sets are proposed.
\end{abstract}

Keywords: Interval-valued intuitionistic fuzzy set, Intuitionistic fuzzy modal operators.

2010 Mathematics Subject Classification: $03 E 72$.

In memory of my father Todor A. Atanassov (1928-1985) who would have been celebrating his 90th birthday today.

\section{Introduction}

Interval-Valued Intuitionistic Fuzzy Sets (IVIFSs; see, e.g., [2]) were introduced as extensions of Intuitionistic Fuzzy Sets (IFSs; see [1,2]) by George Gargov (7.4.1947 - 9.11.1996) and the author in [4]. A lot of operations, relations and operators were defined over them. 
In recent years, the interest in IVIFSs increased and now they are object of intensive research and applications.

A of 2018, the author has started working actively over the development of the IVIFSs theory. In [3], new operators from standard (first) modal type were introduced and some of their basic properties were discussed.

In the present paper, using the notations from paper [3], we introduce a series of modal type operators of second type over IVIFSs. They are analogues of the modal type operators of second type over IFSs, discussed in [2].

\section{Preliminaries}

An IVIFS $A$ over universe $E$ is an object of the form:

$$
A=\left\{\left\langle x, M_{A}(x), N_{A}(x)\right\rangle \mid x \in E\right\},
$$

where $M_{A}(x) \subset[0,1]$ and $N_{A}(x) \subset[0,1]$ are intervals and for all $x \in E$ :

$$
\sup M_{A}(x)+\sup N_{A}(x) \leq 1 .
$$

For any two IVIFSs $A$ and $B$ the following relations hold:

$$
\begin{aligned}
A \subset B \quad \text { iff } \quad & (\forall x \in E)\left(\inf M_{A}(x) \leq \inf M_{B}(x) \& \inf N_{A}(x) \geq \inf N_{B}(x)\right. \\
& \left.\& \sup M_{A}(x) \leq \sup M_{B}(x) \& \sup N_{A}(x) \geq \sup N_{B}(x)\right) \\
A=B \quad \text { iff } \quad & A \subset B \& B \subset A .
\end{aligned}
$$

For any two IVIFSs $A$ and $B$ the following operations hold:

$$
\begin{aligned}
\neg A= & \left\{\left\langle x, N_{A}(x), M_{A}(x)\right\rangle \mid x \in E\right\}, \\
A \cap B= & \left\{\left\langlex,\left[\min \left(\inf M_{A}(x), \inf M_{B}(x)\right), \min \left(\sup M_{A}(x), \sup M_{B}(x)\right)\right],\right.\right. \\
& {\left.\left.\left[\max \left(\inf N_{A}(x), \inf N_{B}(x)\right), \max \left(\sup N_{A}(x), \sup N_{B}(x)\right)\right]\right\rangle \mid x \in E\right\}, } \\
A \cup B= & \left\{\left\langlex,\left[\max \left(\inf M_{A}(x), \inf M_{B}(x)\right), \max \left(\sup M_{A}(x) \sup M_{B}(x)\right)\right],\right.\right. \\
& {\left.\left.\left[\min \left(\inf N_{A}(x), \inf N_{B}(x)\right), \min \left(\sup N_{A}(x), \sup N_{B}(x)\right)\right]\right\rangle \mid x \in E\right\} } \\
A+B= & \left\{\left\langlex,\left[\inf M_{A}(x)+\inf M_{B}(x)-\inf M_{A}(x) \cdot \inf M_{B}(x),\right.\right.\right. \\
& \left.\sup M_{A}(x)+\sup M_{B}(x)-\sup M_{A}(x) \cdot \sup M_{B}(x)\right], \\
& {\left.\left.\left[\inf N_{A}(x) \cdot \inf N_{B}(x), \sup N_{A}(x) \cdot \sup N_{B}(x)\right]\right\rangle \mid x \in E\right\} } \\
A . B= & \left\{\left\langlex,\left[\inf M_{A}(x) \cdot \inf M_{B}(x), \sup M_{A}(x) \cdot \sup M_{B}(x)\right],\right.\right. \\
& {\left[\inf N_{A}(x)+\inf N_{B}(x)-\inf N_{A}(x) \cdot \inf N_{B}(x),\right.} \\
& \left.\left.\left.\sup N_{A}(x)+\sup N_{B}(x)-\sup N_{A}(x) \cdot \sup N_{B}(x)\right]\right\rangle \mid x \in E\right\} \\
= & \left\{\left\langlex,\left[\left(\inf M_{A}(x)+\inf M_{B}(x)\right) / 2,\left(\sup M_{A}(x)+\sup M_{B}(x)\right) / 2\right],\right.\right. \\
& {\left.\left.\left[\left(\inf N_{A}(x)+\inf N_{B}(x)\right) / 2,\left(\sup N_{A}(x)\right\rangle+\sup N_{B}(x)\right) / 2\right] \mid x \in E\right\} }
\end{aligned}
$$


Operators of modal type are defined similarly to those, defined for IFSs:

$$
\begin{aligned}
& \square A=\left\{\left\langle x, M_{A}(x),\left[\inf N_{A}(x), 1-\sup M_{A}(x)\right]\right\rangle \mid x \in E\right\}, \\
& \diamond A=\left\{\left\langle x,\left[\inf M_{A}(x), 1-\sup N_{A}(x)\right], N_{A}(x)\right\rangle \mid x \in E\right\}, \\
& D_{\alpha}(A)=\left\{\left\langlex,\left[\inf M_{A}(x), \sup M_{A}(x)+\alpha \cdot\left(1-\sup M_{A}(x)-\sup N_{A}(x)\right)\right],\right.\right. \\
& \left.\left[\inf N_{A}(x), \sup N_{A}(x)+(1-\alpha) \cdot\left(1-\sup M_{A}(x)-\sup N_{A}(x)\right)\right]\right\rangle \\
& \mid x \in E\} \text {, } \\
& F_{\alpha, \beta}(A)=\left\{\left\langlex,\left[\inf M_{A}(x), \sup M_{A}(x)+\alpha \cdot\left(1-\sup M_{A}(x)-\sup N_{A}(x)\right)\right],\right.\right. \\
& \left.\left[\inf N_{A}(x), \sup N_{A}(x)+\beta \cdot\left(1-\sup M_{A}(x)-\sup N_{A}(x)\right)\right]\right\rangle \\
& \mid x \in E\} \text {, for } \alpha+\beta \leq 1 \text {, } \\
& G_{\alpha, \beta}(A)=\left\{\left\langle x,\left[\alpha \cdot \inf M_{A}(x), \alpha \cdot \sup M_{A}(x)\right],\left[\beta \cdot \inf N_{A}(x), \beta \cdot \sup N_{A}(x)\right]\right\rangle\right. \\
& \mid x \in E\} \text {, } \\
& H_{\alpha, \beta}(A)=\left\{\left\langlex,\left[\alpha \cdot \inf M(x), \alpha \cdot \sup M_{A}(x)\right],\left[\inf N_{A}(x), \sup N_{A}(x)\right.\right.\right. \\
& \left.\left.\left.+\beta .\left(1-\sup M_{A}(x)-\sup N_{A}(x)\right)\right]\right\rangle \mid x \in E\right\}, \\
& H_{\alpha, \beta}^{*}(A)=\left\{\left\langlex,\left[\alpha \cdot \inf M_{A}(x), \alpha \cdot \sup M_{A}(x)\right],\left[\inf N_{A}(x), \sup N_{A}(x)\right.\right.\right. \\
& \left.\left.\left.+\beta \cdot\left(1-\alpha \cdot \sup M_{A}(x)-\sup N_{A}(x)\right)\right]\right\rangle \mid x \in E\right\}, \\
& J_{\alpha, \beta}(A)=\left\{\left\langlex,\left[\inf M_{A}(x), \sup M_{A}(x)+\alpha \cdot\left(1-\sup M_{A}(x)\right.\right.\right.\right. \\
& \left.\left.\left.\left.-\sup N_{A}(x)\right)\right],\left[\beta \cdot \inf N_{A}(x), \beta \cdot \sup N_{A}(x)\right]\right\rangle \mid x \in E\right\}, \\
& J_{\alpha, \beta}^{*}(A)=\left\{\left\langlex,\left[\inf M_{A}(x), \sup M_{A}(x)+\alpha \cdot\left(1-\sup M_{A}(x)\right.\right.\right.\right. \\
& \left.\left.\left.\left.-\beta \cdot \sup N_{A}(x)\right)\right],\left[\beta \cdot \inf N_{A}(x), \beta \cdot \sup N_{A}(x)\right]\right\rangle \mid x \in E\right\},
\end{aligned}
$$

where $\alpha, \beta \in[0,1]$.

The level operators, defined over IVIFS $A$, are defined for $\alpha, \beta \in[0,1]$ and $\alpha+\beta \leq 1$ by:

$$
\begin{aligned}
P_{\alpha, \beta}(A)= & \left\{\left\langlex,\left[\max \left(\alpha, \inf M_{A}(x)\right), \max \left(\alpha, \sup M_{A}(x)\right)\right],\right.\right. \\
& {\left.\left.\left[\min \left(\beta, \inf N_{A}(x)\right), \min \left(\beta, \sup N_{A}(x)\right)\right]\right\rangle \mid x \in E\right\}, } \\
Q_{\alpha, \beta}(A)= & \left\{\left\langlex,\left[\min \left(\alpha, \inf M_{A}(x)\right), \min \left(\alpha, \sup M_{A}(x)\right)\right],\right.\right. \\
& {\left.\left.\left[\max \left(\beta, \inf N_{A}(x)\right), \max \left(\beta, \sup N_{A}(x)\right)\right]\right\rangle \mid x \in E\right\} . }
\end{aligned}
$$

The standard interval valued intuitionistic fuzzy topological operators are defined over each IVIFS $A$ by:

$$
\begin{aligned}
& C(A)=\left\{\left\langle x,\left[\sup _{x \in E} \inf M_{A}(x), \sup _{x \in E} \sup M_{A}(x)\right],\left[\inf _{x \in E} \inf N_{A}(x), \inf _{x \in E} \sup N_{A}(x)\right]\right\rangle \mid x \in E\right\}, \\
& I(A)=\left\{\left\langle x,\left[\inf _{x \in E} \inf M_{A}(x), \inf _{x \in E} \sup M_{A}(x)\right],\left[\sup _{x \in E} \inf N_{A}(x), \sup _{x \in E} \sup N_{A}(x)\right]\right\rangle \mid x \in E\right\} .
\end{aligned}
$$

\section{Main results}

We describe standard interval-valued forms of some intuitionistic fuzzy modal-like operators of second type, following [2], where their IFS-forms are given. We study some basis properties of 
the defined operators. Below, we formulate only these operator properties that are valid for the currently discussed type of operators and are not valid for the next operator extensions.

Let the IVIFS

$$
A=\left\{\left\langle x,\left[\inf M_{A}(x), \sup M_{A}(x)\right],\left[\inf N_{A}(x), \sup N_{A}(x)\right]\right\rangle \mid x \in E\right\},
$$

be given.

Now, we introduce the first two new IVIFS-operators of second type $\boxplus$ and $\bigotimes$ as follows.

$$
\begin{aligned}
& \boxplus A=\left\{\left\langle x,\left[\frac{\inf M_{A}(x)}{2}, \frac{\sup M_{A}(x)}{2}\right],\left[\frac{\inf N_{A}(x)+1}{2}, \frac{\sup N_{A}(x)+1}{2}\right]\right\rangle \mid x \in E\right\}, \\
& 凶 A=\left\{\left\langle x,\left[\frac{\inf M_{A}(x)+1}{2}, \frac{\sup M_{A}(x)+1}{2}\right],\left[\frac{\inf N_{A}(x)}{2}, \frac{\sup N_{A}(x)}{2}\right]\right\rangle \mid x \in E\right\} .
\end{aligned}
$$

All of their properties are valid for their immediare extensions, that for a given real number $\alpha \in[0,1]$ and IVIFS $A$ have the forms:

$$
\begin{aligned}
\boxplus_{\alpha} A= & \left\{\left\langlex,\left[\alpha \inf M_{A}(x), \alpha \sup M_{A}(x)\right],\right.\right. \\
& {\left.\left.\left[\alpha \inf N_{A}(x)+1-\alpha, \alpha \sup N_{A}(x)+1-\alpha\right]\right\rangle \mid x \in E\right\}, } \\
\bigotimes_{\alpha} A= & \left\{\left\langlex,\left[\alpha \inf M_{A}(x)+1-\alpha, \alpha \sup M_{A}(x)+1-\alpha\right],\right.\right. \\
& {\left.\left.\left[\alpha \inf N_{A}(x), \alpha \sup N_{A}(x)\right]\right\rangle \mid x \in E\right\} . }
\end{aligned}
$$

Obviously, for every IVIFS $A$ :

$$
\begin{aligned}
& \boxplus_{0.5} A=\boxplus A, \\
& \bigotimes_{0.5} A=\bigotimes A .
\end{aligned}
$$

Therefore, the new operators $\boxplus_{\alpha}$ and $\bigotimes_{\alpha}$ are generalizations of the first ones.

The following assertions hold for the first two types of the next operators. We give the proof of only one of them (Theorem 1 (b)), while the rest assertions are proved in the same manner.

Theorem 1. For every IVIFS $A$ and for every $\alpha \in[0,1]$ :

(a) $\boxplus_{\alpha} A \subseteq A \subseteq \bigotimes_{\alpha} A$,

(b) $\neg \boxplus_{\alpha} \neg A=\bigotimes_{\alpha} A$,

(c) $\boxplus_{\alpha} \boxplus_{\alpha} A \subseteq \boxplus_{\alpha} A$,

(d) $\bigotimes_{\alpha} \bigotimes_{\alpha} A \supseteq \bigotimes_{\alpha} A$.

Proof. Let $A$ be an IVIFS and $\alpha \in[0,1]$. Then

$$
\begin{gathered}
\neg \boxplus_{\alpha} \neg A \\
=\neg \boxplus_{\alpha} \neg\left\{\left\langle x,\left[\inf M_{A}(x), \sup M_{A}(x)\right],\left[\inf N_{A}(x), \sup N_{A}(x)\right]\right\rangle \mid x \in E\right\} \\
=\neg \boxplus_{\alpha}\left\{\left\langle x,\left[\inf N_{A}(x), \sup N_{A}(x)\right],\left[\inf M_{A}(x), \sup M_{A}(x)\right]\right\rangle \mid x \in E\right\} \\
=\neg\left\{\left\langle x,\left[\alpha \inf N_{A}(x), \alpha \sup N_{A}(x)\right],\left[\alpha \inf M_{A}(x)+1-\alpha, \alpha \sup M_{A}(x)+1-\alpha\right]\right\rangle \mid x \in E\right\}, \\
=\left\{\left\langle x,\left[\alpha \inf M_{A}(x)+1-\alpha, \alpha \sup M_{A}(x)+1-\alpha\right],\left[\alpha \inf N_{A}(x), \alpha \sup N_{A}(x)\right]\right\rangle \mid x \in E\right\} \\
=\bigotimes_{\alpha} A .
\end{gathered}
$$


Theorem 2. For every two IVIFSs $A$ and $B$ :
(a) $\boxplus_{\alpha}(A+B) \subseteq \boxplus_{\alpha} A+\boxplus_{\alpha} B$,
(b) $\bigotimes_{\alpha}(A+B) \supseteq \bigotimes_{\alpha} A+\bigotimes_{\alpha} B$,
(c) $\boxplus_{\alpha}(A . B) \supseteq \boxplus_{\alpha} A . \boxplus_{\alpha} B$,
(d) $\bigotimes_{\alpha}(A . B) \subseteq \bigotimes_{\alpha} A . \bigotimes_{\alpha} B$.

Moreover, the following assertions are also true.

Theorem 3. For every IVIFS $A$ and for every two real numbers $\alpha, \beta \in[0,1]$ :
(a) $\boxplus_{\alpha} \boxplus_{\beta} A=\boxplus_{\beta} \boxplus_{\alpha} A$,
(b) $\bigotimes_{\alpha} \bigotimes_{\beta} A=\bigotimes_{\beta} \rrbracket_{\alpha} A$,
(c) $\bigotimes_{\alpha} \boxplus_{\beta} A \supseteq \boxplus_{\beta} \bigotimes_{\alpha} A$.

Theorem 4. For every IVIFS $A$ and for every three real numbers $\alpha, \beta, \gamma \in[0,1]$ :
(a) $\boxplus_{\alpha} D_{\beta}(A)=D_{\beta}\left(\boxplus_{\alpha} A\right)$,
(b) $\boxplus_{\alpha} F_{\beta, \gamma}(A)=F_{\beta, \gamma}\left(\boxplus_{\alpha} A\right)$, where $\beta+\gamma \leq 1$,
(c) $\boxplus_{\alpha} G_{\beta, \gamma}(A) \subseteq G_{\beta, \gamma}\left(\boxplus_{\alpha} A\right)$,
(d) $\boxplus_{\alpha} H_{\beta, \gamma}(A)=H_{\beta, \gamma}\left(\boxplus_{\alpha} A\right)$,
(e) $\boxplus_{\alpha} H_{\beta, \gamma}^{*}(A)=H_{\beta, \gamma}^{*}\left(\boxplus_{\alpha} A\right)$,
(f) $\boxplus_{\alpha} J_{\beta, \gamma}(A)=J_{\beta, \gamma}\left(\boxplus_{\alpha} A\right)$,
(g) $\boxplus_{\alpha} J_{\beta, \gamma}^{*}(A)=J_{\beta, \gamma}^{*}\left(\boxplus_{\alpha} A\right)$,
(h) $\bigotimes_{\alpha} D_{\beta}(A)=D_{\beta}\left(\bigotimes_{\alpha} A\right)$,
(i) $\bigotimes_{\alpha} F_{\beta, \gamma}(A)=F_{\beta, \gamma}\left(\bigotimes_{\alpha} A\right)$, where $\beta+\gamma \leq 1$,
(j) $\bigotimes_{\alpha} G_{\beta, \gamma}(A) \subseteq G_{\beta, \gamma}\left(\bigotimes_{\alpha} A\right)$,
(k) $\bigotimes_{\alpha} H_{\beta, \gamma}(A)=H_{\beta, \gamma}\left(\bigotimes_{\alpha} A\right)$,
(l) $\bigotimes_{\alpha} H_{\beta, \gamma}^{*}(A)=H_{\beta, \gamma}^{*}\left(\bigotimes_{\alpha} A\right)$,
(m) $\bigotimes_{\alpha} J_{\beta, \gamma}(A)=J_{\beta, \gamma}\left(\bigotimes_{\alpha} A\right)$,
(n) $\bigotimes_{\alpha} J_{\beta, \gamma}^{*}(A)=J_{\beta, \gamma}^{*}\left(\bigotimes_{\alpha} A\right)$. 
The second extension has the forms:

$\boxplus_{\alpha, \beta} A=\left\{\left\langle x,\left[\alpha \inf M_{A}(x), \alpha \sup M_{A}(x)\right],\left[\alpha \inf N_{A}(x)+\beta, \alpha \sup N_{A}(x)+\beta\right]\right\rangle \mid x \in E\right\}$,

$\bigotimes_{\alpha, \beta} A=\left\{\left\langle x,\left[\alpha \inf M_{A}(x)+\beta, \alpha \sup M_{A}(x)+\beta\right],\left[\alpha \inf N_{A}(x), \alpha \sup N_{A}(x)\right]\right\rangle \mid x \in E\right\}$, where $\alpha, \beta, \alpha+\beta \in[0,1]$.

Obviously, for every IVIFS $A$ :

$$
\begin{aligned}
& \boxplus A=\boxplus A_{0.5,0.5}, \\
& \rrbracket A=\bigotimes A_{0.5,0.5}, \\
& \boxplus A_{\alpha}=\boxplus A_{\alpha, 1-\alpha}, \\
& \bigotimes A_{\alpha}=\bigotimes A_{\alpha, 1-\alpha} .
\end{aligned}
$$

The following assertions hold for the new operators.

Theorem 5. For every IVIFS $A$ and for every $\alpha, \beta, \alpha+\beta \in[0,1]$ :

(a) $\neg \boxplus_{\alpha, \beta} \neg A=\bigotimes_{\alpha, \beta} A$,

(b) $\neg \bigotimes_{\alpha, \beta} \neg A=\boxplus_{\alpha, \beta} A$.

Theorem 6. For every IFS $A$ and for every $\alpha, \beta, \gamma, \delta \in[0,1]$ such that $\alpha+\beta, \gamma+\delta \in[0,1]$ :

$$
\boxplus_{\alpha, \beta} \bigotimes_{\gamma, \delta} A \subseteq \bigotimes_{\gamma, \delta} \boxplus_{\alpha, \beta} A
$$

Now, we introduce the third extension of the above operators. They have the forms:

$$
\begin{aligned}
& \bigoplus_{\alpha, \beta, \gamma} A=\left\{\left\langle x,\left[\alpha \inf M_{A}(x), \alpha \sup M_{A}(x)\right],\left[\beta \inf N_{A}(x)+\gamma, \beta \sup N_{A}(x)+\gamma\right]\right\rangle \mid x \in E\right\}, \\
& \bigotimes_{\alpha, \beta, \gamma} A=\left\{\left\langle x,\left[\alpha \inf M_{A}(x)+\gamma, \alpha \sup M_{A}(x)+\gamma\right],\left[\beta \inf N_{A}(x), \beta \sup N_{A}(x)\right]\right\rangle \mid x \in E\right\},
\end{aligned}
$$

where $\alpha, \beta, \gamma \in[0,1]$ and $\max (\alpha, \beta)+\gamma \leq 1$.

Obviously, for every IVIFS $A$ :

$$
\begin{aligned}
& \boxplus A=\boxplus A_{0.5,0.5,0.5}, \\
& \bigotimes A=\bigotimes A_{0.5,0.5,0.5,}, \\
& \boxplus A_{\alpha}=\boxplus A_{\alpha, \alpha, 1-\alpha}, \\
& \bigotimes A_{\alpha}=\bigotimes A_{\alpha, 1-\alpha}, \\
& \boxplus A_{\alpha, \beta}=\boxplus A_{\alpha, \alpha, \beta}, \\
& \bigotimes A_{\alpha, \beta}=\bigotimes A_{\alpha, \alpha, \beta} .
\end{aligned}
$$

The following assertions hold for the new operators.

Theorem 7. For every IVIFS $A$ and for every $\alpha, \beta, \gamma \in[0,1]$, for which $\max (\alpha, \beta)+\gamma \leq 1$ :

(a) $\neg \boxplus_{\alpha, \beta, \gamma} \neg A=\bigotimes_{\beta, \alpha, \gamma} A$,

(b) $\neg \bigotimes_{\alpha, \beta, \gamma} \neg A=\boxplus_{\beta, \alpha, \gamma} A$. 
Theorem 8. For every two IVIFSs $A$ and $B$ and for every $\alpha, \beta, \gamma \in[0,1]$, for which $\max (\alpha, \beta)+$ $\gamma \leq 1$ :
(a) $\boxplus_{\alpha, \beta, \gamma}(A \cap B)=\boxplus_{\alpha, \beta, \gamma} A \cap \boxplus_{\alpha, \beta, \gamma} B$,
(b) $\bigotimes_{\alpha, \beta, \gamma}(A \cap B)=\bigotimes_{\alpha, \beta, \gamma} A \cap \bigotimes_{\alpha, \beta, \gamma} B$,
(c) $\boxplus_{\alpha, \beta, \gamma}(A \cup B)=\boxplus_{\alpha, \beta, \gamma} A \cup \boxplus_{\alpha, \beta, \gamma} B$,
(d) $\bigotimes_{\alpha, \beta, \gamma}(A \cup B)=\bigotimes_{\alpha, \beta, \gamma} A \cup \bigotimes_{\alpha, \beta, \gamma} B$,
(e) $\boxplus_{\alpha, \beta, \gamma}(A @ B)=\boxplus_{\alpha, \beta, \gamma} A @ \boxplus_{\alpha, \beta, \gamma} B$,
(f) $\bigotimes_{\alpha, \beta, \gamma}(A @ B)=\bigotimes_{\alpha, \beta, \gamma} A @ \bigotimes_{\alpha, \beta, \gamma} B$.

Theorem 9. For every IVIFS $A$ and for every $\alpha, \beta, \gamma \in[0,1]$, for which $\max (\alpha, \beta)+\gamma \leq 1$ :

(a) $\boxplus_{\alpha, \beta, \gamma} C(A)=C\left(\boxplus_{\alpha, \beta, \gamma} A\right)$,

(b) $\bigotimes_{\alpha, \beta, \gamma} C(A)=C\left(\bigotimes_{\alpha, \beta, \gamma} A\right)$,

(c) $\boxplus_{\alpha, \beta, \gamma} I(A)=I\left(\boxplus_{\alpha, \beta, \gamma} A\right)$,

(d) $\bigotimes_{\alpha, \beta, \gamma} I(A)=I\left(\bigotimes_{\alpha, \beta, \gamma} A\right)$.

A natural extension of the last two operators $\left(\boxplus_{\alpha, \beta, \gamma}\right.$ and $\left.\bigotimes_{\alpha, \beta, \gamma}\right)$ is the operator

$$
\begin{gathered}
\bullet_{\alpha, \beta, \gamma, \delta} A=\left\{\left\langlex,\left[\alpha \inf M_{A}(x)+\gamma, \alpha \sup M_{A}(x)+\gamma\right],\right.\right. \\
\left.\left.\left[\beta \inf N_{A}(x)+\delta, \beta \sup N_{A}(x)+\delta\right]\right\rangle \mid x \in E\right\},
\end{gathered}
$$

where $\alpha, \beta, \gamma, \delta \in[0,1]$ and $\max (\alpha, \beta)+\gamma+\delta \leq 1$.

It is the fourth type of operators from the current type.

Obviously, for every IVIFS $A$ :

$$
\begin{aligned}
& \boxplus A=\bullet A_{0.5,0.5,0,0.5,}, \\
& \bigotimes A=\bullet A_{0.5,0.5,0.5,0}, \\
& \boxplus A_{\alpha}=\bullet A_{\alpha, \alpha, 0,1-\alpha}, \\
& 凶 A_{\alpha}=\bullet A_{\alpha, \alpha, 1-\alpha, 0}, \\
& \boxplus A_{\alpha, \beta}=\bullet A_{\alpha, \alpha, 0, \beta}, \\
& \bigotimes A_{\alpha, \beta}=\bullet A_{\alpha, \alpha, \beta, 0} . \\
& \boxplus A_{\alpha, \beta, \gamma}=\bullet A_{\alpha, \beta, 0, \gamma}, \\
& \bigotimes A_{\alpha, \beta, \gamma}=\bullet A_{\alpha, \beta, \gamma, 0} .
\end{aligned}
$$

The following assertions hold for the new operator.

Theorem 10. For every IFS $A$ and for every $\alpha, \beta, \gamma, \delta \in[0,1]$, for which

$$
\max (\alpha, \beta)+\gamma+\delta \leq 1:
$$


(a) $\neg \bullet_{\alpha, \beta, \gamma, \delta} \neg A=\bullet_{\beta, \alpha, \delta, \gamma} A$,

(b) $\bullet_{\alpha, \beta, \gamma, \delta}\left(\bullet_{\varepsilon, \zeta, \eta, \theta} A\right)=\bullet_{\alpha \varepsilon, \beta \zeta, \alpha \eta+\gamma, \beta \theta+\delta} A$,

(c) $\bullet_{\alpha, \beta, \gamma, \delta} \square A \supseteq \square \bullet_{\alpha, \beta, \gamma, \delta} A$,

(d) $\bullet_{\alpha, \beta, \gamma, \delta} \diamond A \subseteq \diamond \bullet_{\alpha, \beta, \gamma, \delta} A$.

Theorem 11. For every two IFSs $A$ and $B$ and for every $\alpha, \beta, \gamma, \delta \in[0,1]$, for which

$$
\max (\alpha, \beta)+\gamma+\delta \leq 1:
$$

(a) $\bullet_{\alpha, \beta, \gamma, \delta}(A \cap B)=\bullet_{\alpha, \beta, \gamma, \delta} A \cap \bullet_{\alpha, \beta, \gamma, \delta} B$,

(b) $\bullet_{\alpha, \beta, \gamma, \delta}(A \cup B)=\bullet_{\alpha, \beta, \gamma, \delta} A \cup \bullet_{\alpha, \beta, \gamma, \delta} B$,

(c) $\bullet_{\alpha, \beta, \gamma, \delta}(A @ B)=\bullet_{\alpha, \beta, \gamma, \delta} A @ \bullet_{\alpha, \beta, \gamma, \delta} B$.

In a series of papers (see, e.g., [5,6]), Gökhan Çuvalcioğlu introduced other operators from the second type, but having different forms. The author supposes that he will give for them interval-valued interpretations and for this reason, here the author will not discuss them.

Theorem 12: For every IVIFS $A$ and for every $\alpha, \beta, \gamma, \delta \in[0,1]$, for which

$$
\max (\alpha, \beta)+\gamma+\delta \leq 1:
$$

(a) $\bullet_{\alpha, \beta, \gamma, \delta} C(A)=C\left(\bullet_{\alpha, \beta, \gamma, \delta} A\right)$,

(b) $\bullet_{\alpha, \beta, \gamma, \delta} I(A)=I\left(\bullet_{\alpha, \beta, \gamma, \delta} A\right)$,

(c) $\emptyset_{\alpha, \beta, \gamma, \delta} P_{\varepsilon, \zeta}((A))=P_{\alpha \varepsilon+\gamma, \beta \zeta+\delta}\left(\bullet_{\alpha, \beta, \gamma, \delta} A\right)$,

(d) $\bullet_{\alpha, \beta, \gamma, \delta} P_{\varepsilon, \zeta}((A))=Q_{\alpha \varepsilon+\gamma, \beta \zeta+\delta}\left(\bullet_{\alpha, \beta, \gamma, \delta} A\right)$.

The extended form of all above operators is the operator

$$
\begin{gathered}
\bigcirc_{\alpha, \beta, \gamma, \delta, \varepsilon, \zeta} A=\left\{\left\langlex,\left[\alpha \inf M_{A}(x)-\varepsilon \inf N_{A}(x)+\gamma, \alpha \sup M_{A}(x)-\varepsilon \inf N_{A}(x)+\gamma\right],\right.\right. \\
\left.\left.\left[\beta \inf N_{A}(x)-\zeta \inf M_{A}(x)+\delta, \beta \sup N_{A}(x)-\zeta \inf M_{A}(x)+\delta\right]\right\rangle \mid x \in E\right\},
\end{gathered}
$$

where $\alpha, \beta, \gamma, \delta, \varepsilon, \zeta \in[0,1]$, and

$$
\begin{aligned}
& \max (\alpha-\zeta, \beta-\varepsilon)+\gamma+\delta \leq 1, \\
& \min (\alpha-\zeta, \beta-\varepsilon)+\gamma+\delta \geq 0 .
\end{aligned}
$$

Obviously, for every IVIFS $A$ :

$$
\begin{aligned}
& \boxplus A=\bigcirc_{0.5,0.5,0,0.5,0,0} A, \\
& \bigotimes A=\bigcirc_{0.5,0.5,0.5,0,0,0} A, \\
& \boxplus_{\alpha} A=\bigcirc_{\alpha, \alpha, 0,1-\alpha, 0,0} A,
\end{aligned}
$$




$$
\begin{gathered}
\bigotimes_{\alpha} A=\bigoplus_{\alpha, \alpha, 1-\alpha, 0,0,0} A, \\
\bigoplus_{\alpha, \beta} A=\bigoplus_{\alpha, \alpha, 0, \beta, 0,0} A, \\
\bigotimes_{\alpha, \beta} A=\bigoplus_{\alpha, \alpha, \beta, 0,0,0} A, \\
\bigoplus_{\alpha, \beta, \gamma} A=\bigoplus_{\alpha, \beta, 0, \gamma, 0,0} A, \\
\bigotimes_{\alpha, \beta, \gamma} A=\bigoplus_{\alpha, \beta, \gamma, 0,0,0} A, \\
\bullet_{\alpha, \beta, \gamma, \delta} A=\bigoplus_{\alpha, \beta, \gamma, \delta, 0,0} A .
\end{gathered}
$$

The following assertions hold for the operator $\bigoplus_{\alpha, \beta, \gamma, \delta, \varepsilon, \zeta}$.

Theorem 13. For every IVIFS $A$ and for every $\alpha, \beta, \gamma, \delta, \varepsilon, \zeta \in[0,1]$, for which (1) and (2) are valid, the equality

$$
\neg \bigcirc_{\alpha, \beta, \gamma, \delta, \varepsilon, \zeta} \neg A=\bigcirc_{\beta, \alpha, \delta, \gamma, \zeta, \varepsilon} A
$$

holds.

Theorem 14. For every two IVIFSs $A$ and $B$ and for every $\alpha, \beta, \gamma, \delta, \varepsilon, \zeta \in[0,1]$, for which (1) and (2) are valid, the equality

$$
\bigcirc_{\alpha, \beta, \gamma, \delta, \varepsilon, \zeta}(A @ B)=\bigcirc_{\alpha, \beta, \gamma, \delta, \varepsilon, \zeta} A @ \bigcirc_{\alpha, \beta, \gamma, \delta, \varepsilon, \zeta} B
$$

holds.

We must note that equalities

$$
\begin{aligned}
& \bigcirc_{\alpha, \beta, \gamma, \delta, \varepsilon, \zeta}(A \cap B)=\bigoplus_{\alpha, \beta, \gamma, \delta, \varepsilon, \zeta} A \cap \oslash_{\alpha, \beta, \gamma, \delta, \varepsilon, \zeta} B, \\
& \bigcirc_{\alpha, \beta, \gamma, \delta, \varepsilon, \zeta}(A \cup B)=\bigoplus_{\alpha, \beta, \gamma, \delta, \varepsilon, \zeta} A \cup \bigcirc_{\alpha, \beta, \gamma, \delta, \varepsilon, \zeta} B,
\end{aligned}
$$

which are valid for operator $\emptyset_{\alpha, \beta, \gamma, \delta}$, are not always valid now.

Theorem 15. For every IVIFS $A$ and for every $\alpha_{1}, \beta_{1}, \gamma_{1}, \delta_{1}, \varepsilon_{1}, \zeta_{1}, \alpha_{2}, \beta_{2}, \gamma_{2}, \delta_{2}, \varepsilon_{2}, \zeta_{2} \in[0,1]$ for which conditions that are similar to (1) and (2) are valid, the equality

$$
\begin{gathered}
\bigoplus_{\alpha_{1}, \beta_{1}, \gamma_{1}, \delta_{1}, \varepsilon_{1}, \zeta_{1}}\left(\bigcirc_{\alpha_{2}, \beta_{2}, \gamma_{2}, \delta_{2}, \varepsilon_{2}, \zeta_{2}} A\right) \\
=\bigcirc_{\alpha_{1} \cdot \alpha_{2}+\varepsilon_{1} \cdot \zeta_{2}, \beta_{1} \cdot \beta_{2}+\zeta_{1} \cdot \varepsilon_{2}, \alpha_{1} \cdot \gamma_{2}-\varepsilon_{1} \cdot \delta_{2}+\gamma_{1}, \beta_{1} \cdot \delta_{2}-\zeta_{1} \cdot \gamma_{2}+\delta_{1}, \alpha_{1} \cdot \varepsilon_{2}+\varepsilon_{1}, \beta_{2}, \beta_{1} \cdot \zeta_{2}+\zeta_{1} \cdot \alpha_{2} A} A
\end{gathered}
$$

holds.

\section{Conclusion}

In a next paper, the author will introduce generalizations of all the operators, introduced here for a first time. Here, inf- and sup-components have equal parameters (real numbers). In the next research, these parameters for the inf- and sup-components will be different and their specific properties will be studied.

In near future, the author plans to extend the results from the present paper to a book. 


\section{Acknowledgments}

This paper has been partially supported by the Bulgarian National Science Fund under the Grants Ref. No. DN 02/10 "New Instruments for Knowledge Discovery from Data and their Modelling" and Ref. No. DN 17/06 "A New Approach, Based on an Intercriteria Data Analysis, to Support Decision Making in in silico Studies of Complex Biomolecular Systems".

\section{References}

[1] Atanassov, K. (1999) Intuitionistic Fuzzy Sets. Springer, Heidelberg.

[2] Atanassov, K. (2012) On Intuitionistic Fuzzy Sets Theory, Springer, Berlin.

[3] Atanassov, K. (2018) On interval-valued intuitionistic fuzzy modal operators. Notes on Intuitionistic Fuzzy Sets, 24(1), 1-12.

[4] Atanassov, K. \& Gargov, G. (1989) Interval valued intuitionistic fuzzy sets, Fuzzy Sets and Systems, 31(3), 343-349.

[5] Çuvalcioğlu, G. (2007) Some properties of $E_{\alpha, \beta}$ operator. Advanced Studies in Contemporary Mathematics, 14(2), 305-310.

[6] Çuvalcioğlu, G. (2010) Expand the model operator diagram with $Z_{\alpha . \beta}^{\omega}$. Proceedings of the Jangjeon Math. Society, 13(3), 403-412. 